\title{
Recurrent severe aortic stenosis after transfemoral transcatheter valve-in-valve-in-valve replacement
}

\author{
Tomoki Ochiai, MD, Sung-Han Yoon, MD, Rahul Sharma, MD, Tarun Chakravarty, MD, \\ Mamoo Nakamura, MD, Wen Cheng, MD, and Raj Makkar, MD, Los Angeles, Calif
}

\author{
From the Heart Institute, Cedars-Sinai Medical Center, Los Angeles, Calif. \\ Sources of funding: None. \\ Disclosures: R.M. received grant support from Edwards Lifesciences; is a consultant for Abbott Vascular, Cordis \\ Corporation, and Medtronic; and holds equity in Entourage Medical. R.S. is a consultant for Edwards Life- \\ sciences, Abbott Vascular, Keystone Heart, and Boston Scientific. All other authors have nothing to disclose \\ with regard to commercial support. \\ Received for publication Aug 27, 2017; revisions received Nov 16, 2017; accepted for publication Dec 20, 2017; \\ available ahead of print Feb 6, 2018. \\ Address for reprints: Raj Makkar, MD, Advanced Health Sciences Pavilion, Cedars-Sinai Heart Institute, $127 \mathrm{~S}$ \\ San Vicente Blvd, Third Floor, Suite A3421, Los Angeles, CA 90048 (E-mail: Raj.Makkar@cshs.org). \\ J Thorac Cardiovasc Surg 2018;155:e141-4 \\ $0022-5223 / \$ 36.00$ \\ Copyright (C) 2018 by The American Association for Thoracic Surgery \\ https://doi.org/10.1016/j.jtcvs.2017.12.120
}

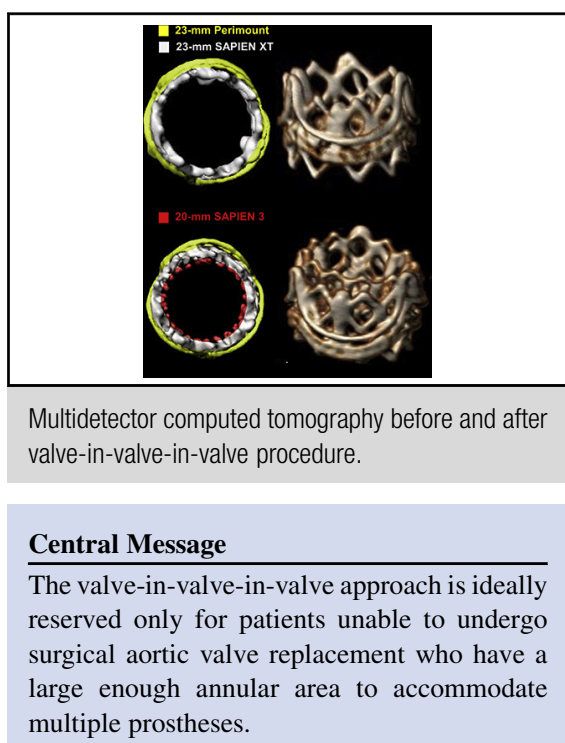

See Editorial Commentary page e145.
Transcatheter aortic valve-in-valve (VIV) replacement within degenerated surgical bioprostheses is a safe and feasible therapeutic alternative to redo surgery. Currently, the main limitation of aortic VIV procedure is the possibility of high postprocedural gradients. ${ }^{1}$ We describe a case of transfemoral transcatheter aortic valve-in-valve-in-valve replacement for high postprocedural gradient after a VIV procedure.

\section{CLINICAL SUMMARY}

A 71-year-old woman with a body surface area of $1.79 \mathrm{~m}^{2}$ presented with progressive shortness of breath. Her surgical history included surgical aortic valve replacement (SAVR) for bacterial endocarditis in 1991, followed by redo SAVR for prosthetic aortic stenosis (AS) in 2004 with a 23-mm Carpentier-Edwards Perimount bioprosthesis (Edwards Lifesciences, Irvine, Calif). In December 2013, severe prosthetic AS (peak and mean gradients of 110 and $67 \mathrm{~mm} \mathrm{Hg}$, respectively, and aortic valve area of $0.3 \mathrm{~cm}^{2}$ ) was diagnosed, and the patient underwent transcatheter VIV replacement with a 23-mm Sapien XT valve (Edwards Lifesciences). The postprocedural transthoracic echocardiogram (TTE) revealed peak and mean gradients of 21 and $13 \mathrm{~mm} \mathrm{Hg}$, respectively, and aortic insufficiency was trivial. Aspirin was continued after the procedure. In April 2016, the patient presented with fatigue, and the TTE demonstrated high transaortic gradients (peak and mean gradients of 122 and $82 \mathrm{mmHg}$, respectively, and aortic valve area of $0.3 \mathrm{~cm}^{2}$ ), consistent with recurrent severe AS after VIV replacement. Although the multidetector computed tomographic scan revealed no hypoattenuated leaflet thickening or reduced leaflet motion (Figure 1, A, and Video 1), anticoagulant therapy with warfarin was started empirically in the context of concern for potential risk of leaflet thrombosis.

Despite anticoagulant therapy for 2 months, the patient had progressive congestive heart failure develop (New York Heart Association functional class III symptoms and brain natriuretic peptide level of $441 \mathrm{pg} / \mathrm{mL}$ ). The TTE showed unchanged prosthetic AS (peak and mean gradients of 114 and $72 \mathrm{~mm} \mathrm{Hg}$, respectively) unresponsive to anticoagulant therapy. She was deemed at high risk if undergoing SAVR because of 2 previous open surgeries and hepatic cirrhosis (Child-Pugh grade A) and was therefore evaluated for transfemoral transcatheter intervention. The multidetector computed tomographic scan demonstrated an aortic annular area of $269.7 \mathrm{~mm}^{2}$, with a mean diameter of $18.6 \mathrm{~mm}$ (Figure 1, $B$ and $C$ ). A 20-mm Sapien 3 valve (Edwards Lifesciences) was selected based on the annular area. This valve was successfully deployed within the previous prostheses (Video 2). The postprocedural TTE demonstrated a reduction in gradients (peak and mean gradients 

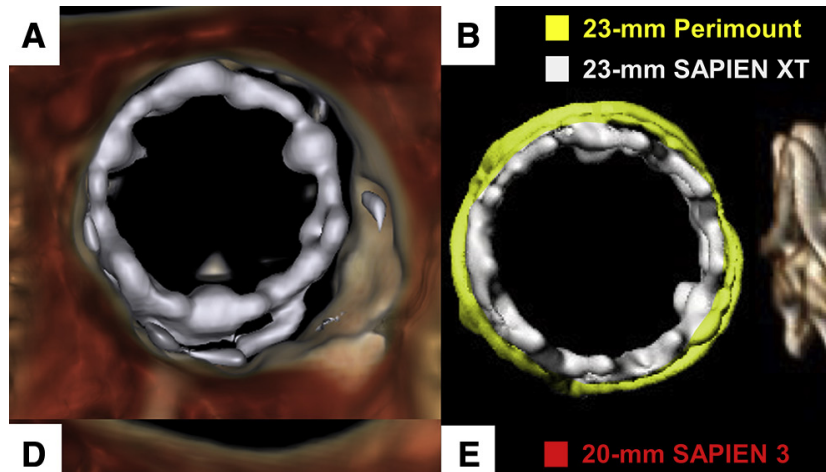

c

Area: $269.7 \mathrm{~mm}^{2}$
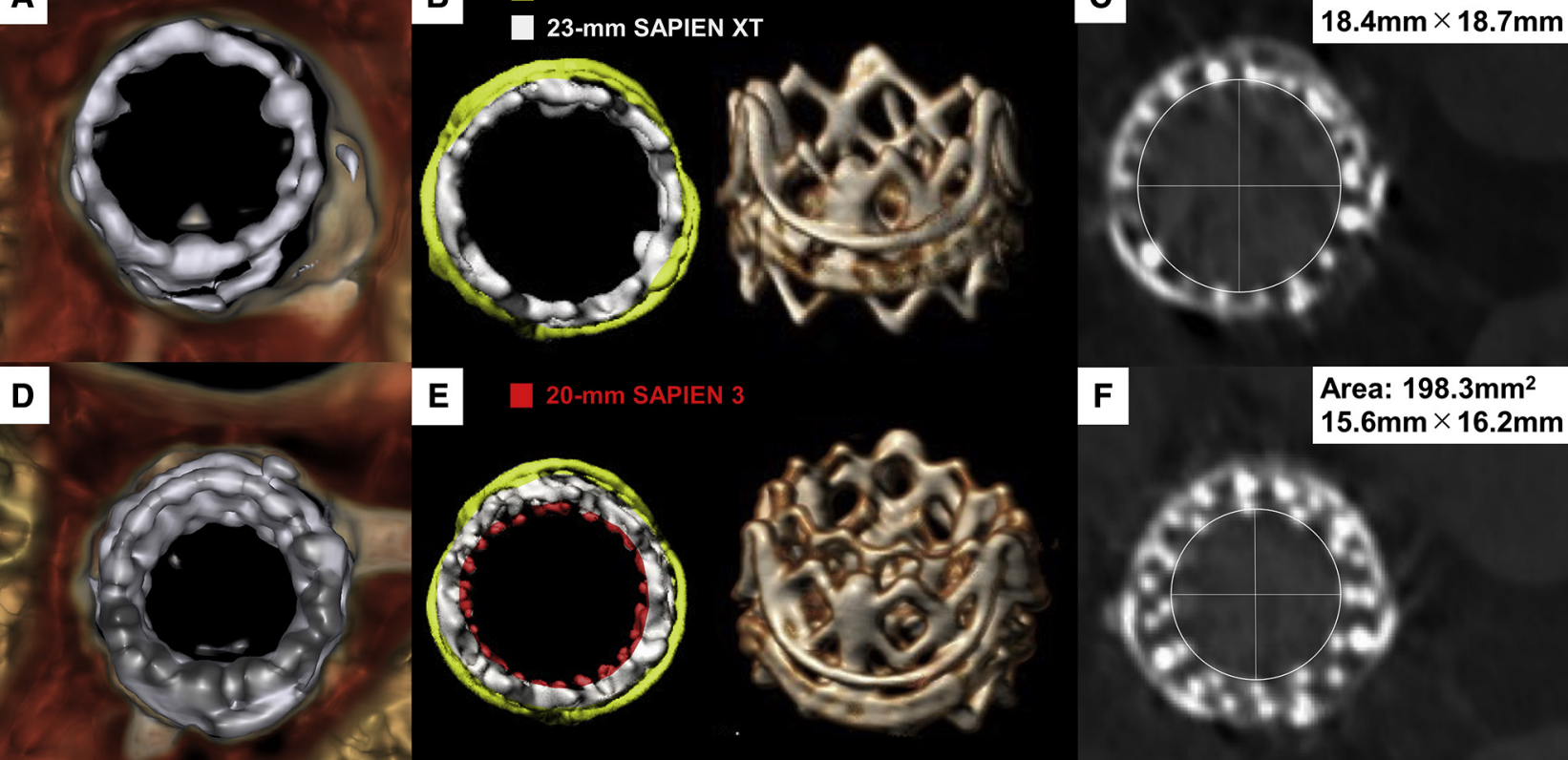

Area: $198.3 \mathrm{~mm}^{2}$ $15.6 \mathrm{~mm} \times 16.2 \mathrm{~mm}$

FIGURE 1. Multidetector computed tomographic scans before and after valve-in-valve-in-valve procedure. A, Multidetector computed tomographic scan before the procedure revealed neither hypoattenuated leaflet thickening nor reduced leaflet motion. B and C, Multidetector computed tomographic scan before the procedure revealed an aortic annular area of $269.7 \mathrm{~mm}^{2}$, with an average diameter of $18.6 \mathrm{~mm}$. D, Multidetector computed tomographic scan after the procedure revealed neither hypoattenuated leaflet thickening nor reduced leaflet motion. E and F, Multidetector computed tomography scan after the procedure revealed an aortic annular area of $198.3 \mathrm{~mm}^{2}$, with an average diameter of $15.9 \mathrm{~mm}$.

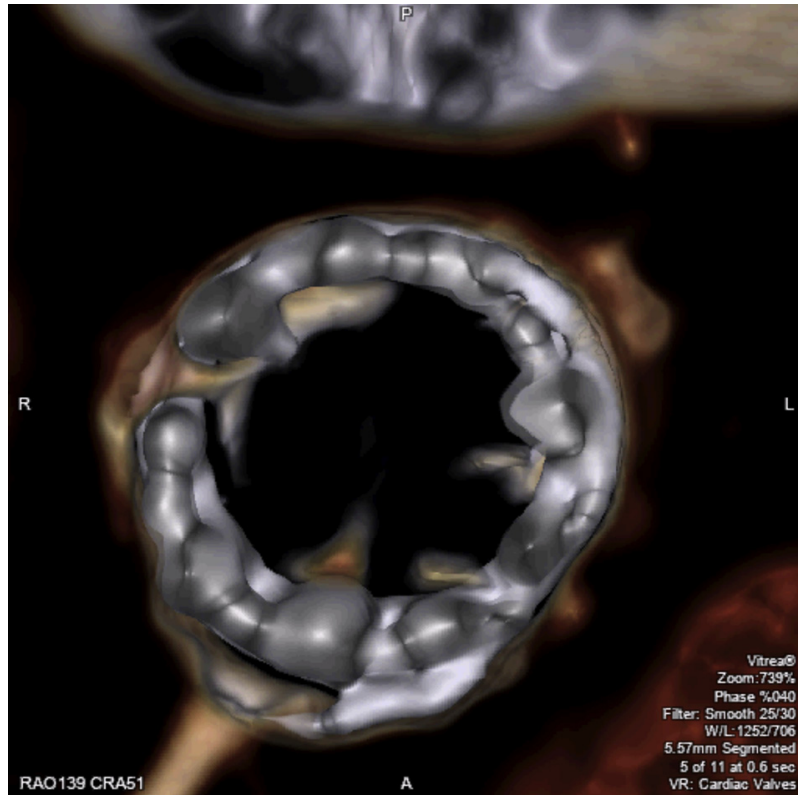

VIDEO 1. Multidetector computed tomographic scan before the valve-invalve-in-valve procedure. Multidetector computed tomographic scan before the procedure revealed neither hypoattenuated leaflet thickening nor reduced leaflet motion. Video available at: http://www.jtcvsonline. org/article/S0022-5223(18)30042-4/fulltext.

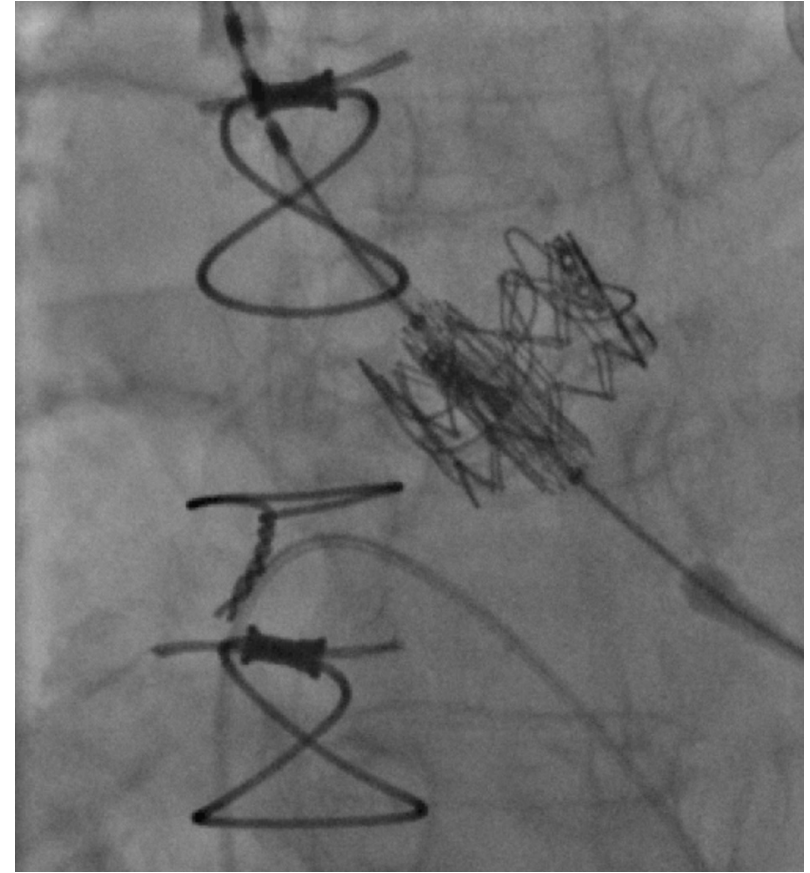

VIDEO 2. Angiogram during valve-in-valve-in-valve deployment. A 20mm Sapien 3 valve (Edwards Lifesciences, Irvine, Calif) was successfully implanted in the valvular complex. Video available at: http://www. jtcvsonline.org/article/S0022-5223(18)30042-4/fulltext. 


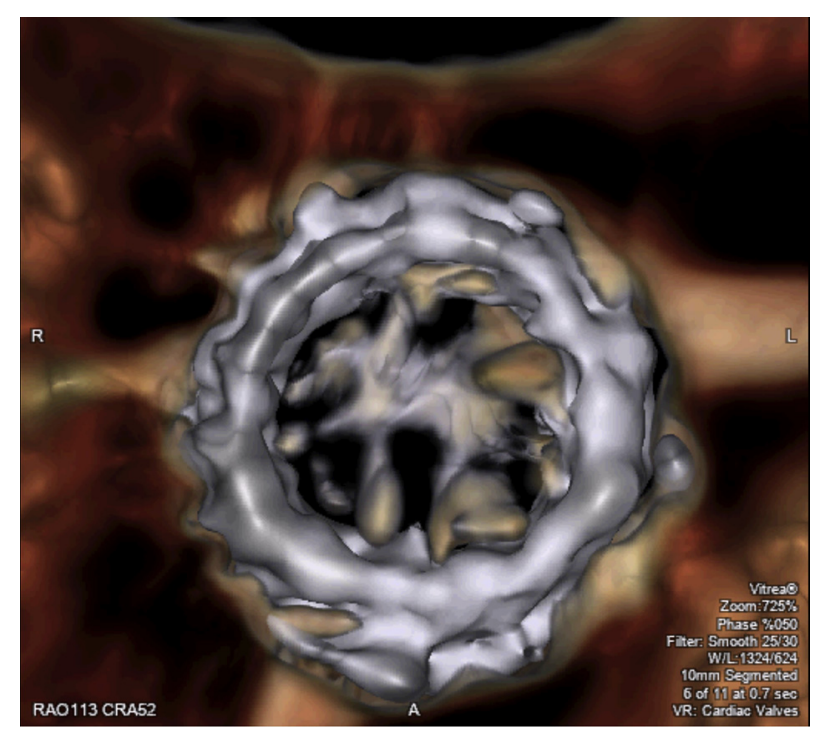

VIDEO 3. Multidetector computed tomographic scan after the valve-invalve-in-valve procedure. Multidetector computed tomography scan after the procedure revealed neither hypoattenuated leaflet thickening nor reduced leaflet motion. Video available at: http://www.jtcvsonline.org/ article/S0022-5223(18)30042-4/fulltext.

of 53 and $31 \mathrm{~mm} \mathrm{Hg}$, respectively); however, a severe prosthesis-patient mismatch (PPM) with an indexed effective orifice area of $0.59 \mathrm{~cm}^{2} / \mathrm{m}^{2}$ was noted.

After 8 months, the patient presented with recurrent symptoms of congestive heart failure. The TTE showed elevated transaortic gradients (peak and mean gradients of 68 and $41 \mathrm{~mm} \mathrm{Hg}$, respectively, and aortic valve area of $0.8 \mathrm{~cm}^{2}$ ). A repeat multidetector computed tomographic scan demonstrated no sign of leaflet thrombosis or reduced leaflet motion (Figure 1, D, and Video 3). The measured aortic annular area was $198.3 \mathrm{~mm}^{2}$, with a mean diameter of $15.9 \mathrm{~mm}$ (Figure 1, $E$ and $F$ ). Although the patient was deemed at high risk if undergoing a third redo sternotomy and SAVR, our heart team considered this to be the most appropriate approach to address the persistent high gradient with significant PPM. During SAVR, after the removal of 3 previously implanted prosthetic valves (1 surgical and 2 transcatheter valves), the annulus was débrided, and a 21$\mathrm{mm}$ pericardial Magna Ease valve (Edwards Lifesciences) was inserted. The pathologic examination after the procedure showed the presence of dense adherent fibrosis and calcification in the 23-mm Carpentier-Edwards Perimount bioprosthesis (Figure 2, $A$ ) and the 23-mm Sapien XT valve (Figure 2, B). No fibrosis or calcification was seen in the 20mm Sapien 3 valve (Figure 2, C). In all 3 valves, no thrombus or vegetation was identified. The patient had an uneventful postoperative course and was discharged on postoperative day 7 .

\section{DISCUSSION}

This is the first case report to document transcatheter valve-in-valve-in-valve replacement to include pathologic and computed tomographic findings after the procedure.

Bioprosthetic surgical valves are increasingly used in preference to mechanical valves in SAVR, particularly in younger populations. This is driven by patient preference for avoidance of anticoagulant therapy and the possibility of future transcatheter VIV implantation. ${ }^{2}$ Although aortic VIV procedure has become an established treatment option for failed surgical bioprostheses, there are still limitations, such as high postprocedural transvalvular gradients. In this case, the pathologic findings revealed that development of recurrent severe AS after the first SAVR and the first transcatheter VIV was caused by structural degeneration of prosthetic valves, with dense adherent fibrosis and calcification. In the second transcatheter heart valve, however, there was no evidence of structural degeneration manifested by impaired leaflet mobility, significant calcification, or pannus. In general, small bioprostheses have small effective orifice areas, which results in higher gradients, even in the absence of structural degeneration. ${ }^{3}$ In this case, severe

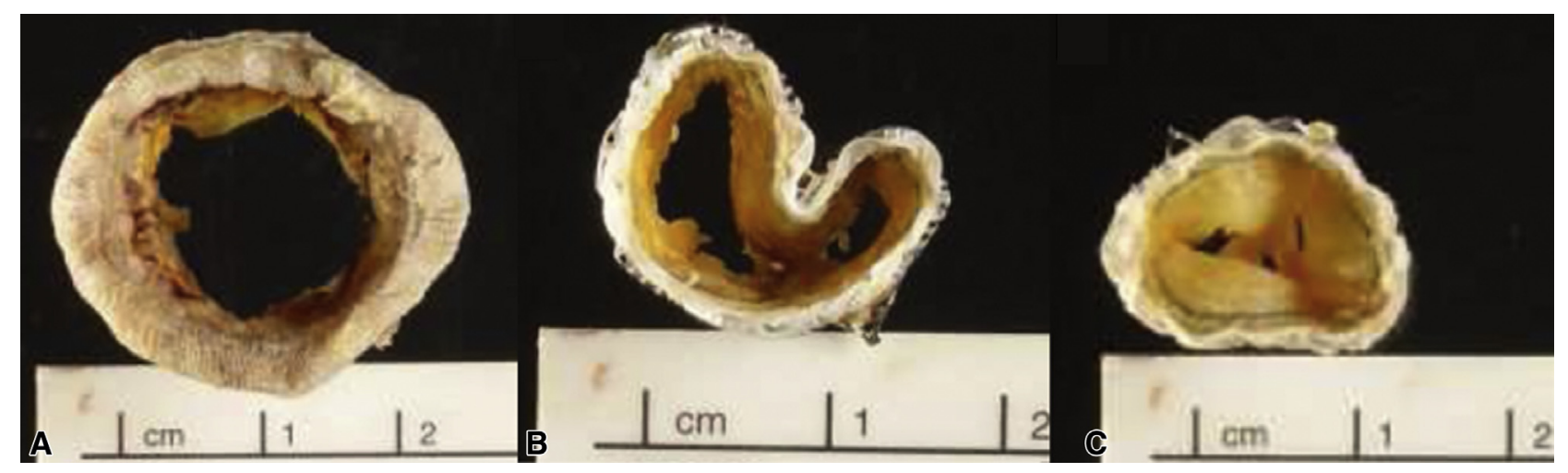

FIGURE 2. Pathologic findings of 3 prosthetic valves during surgical aortic valve replacement. A, Dense adherent fibrosis and calcification in the $23-\mathrm{mm}$ Carpentier-Edwards Perimount bioprosthesis (Edwards Lifesciences, Irvine, Calif). B, Dense adherent fibrosis and calcification in the 23-mm Sapien XT (Edwards Lifesciences). C, No fibrosis, calcification, or thrombus was seen in the 20-mm Sapien 3 prosthesis (Edwards Lifesciences). 
PPM seemed to be the cause of recurring high gradients after the valve-in-valve-in-valve procedure. In light of recent data showing lower gradients after VIV procedures with a supra-annular valve, ${ }^{4}$ the clinical course in this case might have been avoided with the use of a supra-annular transcatheter aortic valve. Treatment with balloon aortic valvuloplasty may be an option, although this is unlikely to provide any sustained benefit. As demonstrated in this case, redo SAVR is the most effective choice to maximize the effective orifice area and mitigate the risk of PPM. Most affected patients are poor candidates for redo SAVR, however, creating a management dilemma.

Treating patients with severe AS after VIV is extremely challenging because of the limited space for transcatheter heart valve expansion inside these valves. Accordingly, the risk of PPM after implantation is high. The valvein-valve-in-valve approach is a rare recourse, ideally reserved only for patients unable to undergo SAVR who have a large enough annular area to accommodate multiple prostheses and avoid PPM. This case raises concerns about repeating transcatheter treatment in patients with elevated gradients with PPM after VIV. In addition, it will be more important for surgeons to make the best use of techniques to provide the largest effective orifice area in the SAVR procedure while paying attention to the intraoperative complications. High postprocedural gradients after VIV will continue to challenge clinicians, particularly in a younger population in which the valves are expected to function for a longer period. For such patients, future studies are warranted to identify the optimal management approaches.

\section{References}

1. Dvir D, Webb JG, Bleiziffer S, Pasic M, Waksman R, Kodali S, et al. Valve-inValve International Data Registry Investigators. Transcatheter aortic valve implantation in failed bioprosthetic surgical valves. JAMA. 2014;312:162-70.

2. Isaacs AJ, Shuhaiber J, Salemi A, Isom OW, Sedrakyan A. National trends in utilization and in-hospital outcomes of mechanical versus bioprosthetic aortic valve replacements. J Thorac Cardiovasc Surg. 2015;149:1262-9.e3.

3. Rosenhek R, Binder T, Maurer G, Baumgartner H. Normal values for Doppler echocardiographic assessment of heart valve prostheses. J Am Soc Echocardiogr. 2003; 16:1116-27.

4. Simonato M, Webb J, Kornowski R, Vahanian A, Frerker C, Nissen H, et al. Transcatheter replacement of failed bioprosthetic valves: large multicenter assessment of the effect of implantation depth on hemodynamics after aortic valve-in-valve. Circ Cardiovasc Interv. 2016;9: https://doi.org/10.1161/CIRC INTERVENTIONS.115.003651 